\title{
¿DE QUÉ SE HABLA CUÁNDO SE HABLA DE POLÍTICAS PÚBLICAS? ESTADO DE LA DISCUSIÓN Y ACTORES EN EL CHILE DEL BICENTENARIO
}

\author{
MireYa DÁvila (mdavila@uahurtado.cl) \\ Universidad Alberto Hurtado, Chile \\ Ximena Soto Soutullo (xsotos@ucentral.cl) \\ Universidad Central, Chile
}

Desde 1990 en Chile el uso del concepto de políticas públicas ha crecido enormemente. Las acciones del Estado para solucionar problemas públicos son objeto de escrutinio por diversos actores académicos y políticos. Estas parecen ser el lenguaje más valorado a la hora de plantear los contenidos del debate público. ¿Qué y quiénes enseñan y discuten políticas públicas en Chile? Este artículo analiza la enseñanza de políticas públicas en pre y post grado y su uso en los principales think tanks. La hipótesis del trabajo es que la economía es la ciencia social que más influye en el uso académico de este enfoque. Esto se explica por el vínculo entre conocimiento experto y economía. Se concluye que a nivel de posgrado, la economía predomina en la enseñanza de políticas públicas y que el uso de este enfoque no es generalizado en los centros de estudios.

Palabras Clave: Politicas Públicas; Economia; Tecnocracia; Posgrado; Think tanks. 


\section{WHEN TALKING ABOUT PUBLIC POLICY, WHAT DO WE REALLY MEAN? ACTORS AND ISSUES IN CHILE}

Since 1990 the use of the concept of public policy has been increasingly used in Chile. State measures aimed at solving public problems are scrutinized by both academic and political actors. Public policy is the most common approach for framing content within a public debate. Who is teaching and discussing public policy? This article offers a preliminary analysis of the way policy is taught in Chilean undergraduate and postgraduate programs as well as its use in the country's main think tanks. We sustain that is economics is the dominant discipline in this approach. This is explained by the relationship between expert knowledge and economics. We conclude that economics dominates postgraduate programs in public policy, while this approach is not generalized among major think tanks.

Keywords: Public policy; Economic; Technocracy; Postgraduate Studies; Think Tanks. 


\section{INTRODUCCIÓN ${ }^{1}$}

Tradicionalmente las políticas públicas han formado parte del conjunto de disciplinas, como la ciencia política, que estudian el Estado y los gobiernos. Lo particular de este enfoque, nacido como una parte de la ciencia política y la administración pública, es la orientación hacia el conocimiento práctico y la solución de problemas públicos en contextos democráticos.

Originado en la década de los cincuenta en Estados Unidos, el estudio de las políticas públicas creció enormemente en la segunda década del siglo pasado. En Chile, la vuelta a la democracia trajo este enfoque como parte de las decisiones y acciones de los gobiernos de la Concertación a partir de la transición a la democracia a comienzos de los años noventa. Común en América Latina y en Occidente en general, el enfoque de políticas públicas ha sido especialmente popular dentro de las élites políticas, de gobierno y en los organismos internacionales que analizan e influyen en la solución de los problemas públicos.

Desde el restablecimiento de la democracia en Chile a inicios de los noventa fue posible observar una relación constante entre conocimiento experto vinculado a la economía y el enfoque de políticas públicas, especialmente en temas sectoriales como manejo macroeconómico, políticas laborales, entre otras (Dávila, 2010, $2011)^{2}$. De hecho es posible, cada cierto tiempo, apreciar un cierto malestar por parte de elites políticas tradicionales que cuestionan la influencia de estos expertos, los llamados tecnócratas, que deciden sobre aspectos tradicionalmente radicados en el mundo de la política³.

1 Agradecemos a Felipe Tello y Serio Pirinoli por sus comentarios y aportes a las versiones preliminares de este artículo. Del mismo modo, reconocemos el aporte de los evaluadores anónimos que revisaron este artículo para su publicación. Finalmente, agradecemos a los estudiantes e investigadores del Observatorio de Gobierno y Políticas Públicas de la Universidad Alberto Hurtado, Maximiliano Prieto, Danilo Miranda, Diego Poblete y Gabriel Guzmán por su apoyo e interés en los temas públicos.

2 De hecho, el núcleo o grupo fundante de la Concertación en 1989 estuvo compuesto por economistas que influyeron decididamente en las políticas económicas (especialmente macroeconómicas) que postuló y luego implementó el entonces candidato concertacionista, Patricio Aylwin.

3 Un ejemplo de lo anterior es la crítica que hizo el ex Ministro de la Concertación Francisco Vidal, quien responsabilizó a los tecnócratas de la Concertación de decisiones que tuvieron altos costos políticos y contribuyeron a la pérdida del gobierno. www.elmostrador.cl 10/6/2010. 
Es a partir de lo anterior, de esta relación entre políticas públicas, conocimiento experto y economía que nos interesa particularmente realizar un diagnóstico general de quiénes están enseñando políticas públicas y quiénes las estudian, para dilucidar entonces cuál es el enfoque predominante. De ese modo, se presentan algunas conclusiones en relación a criterios de soluciones a los problemas públicos y contribuir al debate acerca de la discusión de los temas públicos en la democracia chilena. Por consiguiente, esta investigación se pregunta sobre quién y qué de este enfoque se está aplicando en Chile actualmente.

Por ser este un artículo exploratorio se trabaja a partir de cuestionamientos básicos, derivados del análisis de la relación entre tecnocracia y conocimiento experto en las administraciones concertacionistas (1990 - 2010). Este período resulta atractivo de estudiar porque a partir de 1990 Chile comenzó un nuevo período democrático caracterizado por un nuevo diseño institucional heredado de los militares, expresado principalmente en la Constitución de 1980 (Siavelis, 2000). Destaca también de la democracia chilena post autoritaria la estabilidad de las coaliciones políticas, especialmente de la Concertación como coalición de gobierno. Por otro lado, la Concertación resulta interesante de analizar no sólo por su estabilidad sino también porque ésta reunió partidos y mundos políticos (la izquierda y la Democracia Cristiana) que en las décadas previas al Golpe de 1973 eran enemigos políticos. Si bien esta coalición nació en torno al clivaje democracia - dictadura, no es menos cierto que entre los partidos y al interior de éstos había divisiones ideológicas en cuanto al modelo de libre mercado y las estrategias de continuidad y cambio. Como señala la literatura (Altman, 2000; Amorim Neto, 2003, 2006) las coaliciones políticas no son un fenómeno común en la política latinoamericana y menos la extraordinaria estabilidad mostrada por la Concertación (Chasquetti, 2006). Finalmente, resulta interesante analizar cómo ha evolucionado desde la dictadura la presencia e influencia de la tecnocracia y el conocimiento experto en economía (Huneeus, 1998).

A partir de investigaciones desarrolladas por Dávila $(2010,2011)$ sobre el rol de la tecnocracia en las cuatro administraciones concertacionistas (1990 - 2010), se avanza en diagnosticar si esta influencia de la economía como el conocimiento experto dominante a nivel de gobierno se extiende también a la enseñanza de las políticas públicas en Chile y la discusión en los principales centros de estudios chilenos. Es así entonces que este artículo da una primera mirada respecto 
al conocimiento del enfoque de políticas públicas en Chile, a partir del análisis de las principales instituciones universitarias que están enseñando cursos o post grados sobre políticas públicas y del análisis de los centros de estudios o think tanks.

A partir de las conclusiones de los datos obtenidos en este trabajo se continúa analizando la penetración de las políticas públicas en el análisis de los temas públicos. En una agenda de investigación de largo alcance interesa analizar y explicar cómo y quiénes diseñan las políticas públicas en Chile.

Como primera etapa de un análisis más exhaustivo sobre el vínculo entre conocimiento experto, economía y políticas públicas, se realiza un mapeo general del estado del arte respecto al análisis y uso del concepto de políticas públicas en Chile. Particularmente, este trabajo se centra en dos ámbitos: formación profesional a nivel universitario de pregrado, postgrado e investigación en centros de estudios.

Respecto a lo primero, debido a que no hay carreras de pregrado de políticas públicas, se realiza una revisión de las mallas curriculares de todas las carreras en todas las universidades chilenas, seleccionando sólo aquellas que dicten cursos de políticas públicas en pregrado. Cursos como "políticas sociales", "evaluación de proyectos sociales" u otros similares, quedan excluidos por no contener explícitamente el concepto que se analiza posteriormente. En un segundo paso y con el objetivo de dar cuenta de la existencia de posgrados en políticas públicas, estudiamos sólo aquellos que contengan explícitamente en sus nombres o menciones el concepto de política pública. Aquellos que cuenten con al menos un ramo de política pública, pero no lo expliciten en su grado o nombre comercial, quedaron excluidos del análisis, por cuanto no son considerados como parte de la especialización de políticas públicas a nivel de posgrado.

Como hipótesis de trabajo se postula que el estudio de políticas públicas a nivel de pregrado se da de manera transversal a carreras ligadas a distintas facultades, como por ejemplo economía, educación, ciencias sociales en general. Mientras, que a nivel de posgrado, el estudio de políticas públicas se encuentra mayormente desarrollado y dictado desde facultades de economía o ingeniería.

Lo anterior nos permite a la vez argumentar que el análisis de las políticas públicas se centra primordialmente en un complemento de la ciencia política y que el análisis de los temas sectoriales está dominado por la economía. 
A partir de lo anterior, primeramente se hace una descripción general del concepto y sus características, sus principales orientaciones así como sus aspectos más utilizados. A continuación desarrollamos la relación entre políticas públicas, experticia y economía. Posteriormente se presentan las características principales de la aplicación de este enfoque en Chile, en el área de la enseñanza universitaria y de los principales think tanks. En seguida presentamos el análisis de los datos, una contrastación empírica a partir de lo arrojado de la recolección de información de nuestro estudio de campo. Finalmente, una reflexión en torno a las implicancias de la predominancia del enfoque económico en el estudio de los temas públicos y los futuros temas de investigación.

\section{1. ¿QUÉ SON LAS POLÍTICAS PÚBLICAS?}

El estudio de las políticas públicas tiene como objetivo analizar los problemas públicos, su origen y soluciones en contextos democráticos. Vistas como acciones de los Estados y los gobiernos para solucionar estos problemas, este enfoque se concentra en el cómo y por qué estas instituciones actúan, junto con otros actores políticos, económicos y sociales, para solucionar dichos problemas públicos. El contexto democrático está dado por la necesidad de dar valor a este régimen político cómo único espacio posible para resolución de los temas públicos.

Fue Laswell quien en el libro The Policy Sciences (Laswell y Lerner, 1951) acuñó el término ciencias de política o policy sciences, para denominar el estudio de las políticas. La idea fue que desde las ciencias sociales se desarrollasen métodos para resolver problemas sociales. El objetivo era producir conocimiento científico que contribuyera a mejorar la calidad de vida y corregir los defectos del gobierno democrático. Conocimiento experto en contextos políticos parece resumir la esencia del enfoque de políticas públicas. Cuán experto, científico, neutral o, desde otra perspectiva, cuánto influye la política en este conocimiento de políticas públicas parece estar en el centro del debate sobre cómo abordar el estudio de estas acciones del Estado. Para algunos este conocimiento experto se da en contextos democráticos donde factores políticos también deben ser considerados (Stone, 1997; Schneider e Ingram, 2007). Es así que otros autores enfatizan los temas 
éticos y de negociación política involucrados en la políticas públicas (Susskind, 2006; Merino, 2010).

El estudio de las políticas públicas o policy sciences es un estudio orientado a los problemas $^{4}$, ya que desde una perspectiva científica no es posible argumentar a favor de la validez intrínseca de los valores. El objetivo es, entonces, que los enunciados de la ciencia (el análisis de las políticas públicas) sean una norma técnica para la elección y práctica de políticas o acciones del Estado. Visto de otra forma, este análisis de las políticas públicas debe dar la posibilidad de que la ciencia ayude a ordenar preferencias y metas de actores políticos. Entonces el conocimiento de políticas debe permitir seleccionar políticas de la mejor forma posible (racionalmente hablando). Para otros autores lo anterior no siempre sucede, pues esta racionalidad no siempre se cumple (Andrews, 2007).

El estudio de las políticas públicas ha implicado el estudio de las acciones de los gobiernos y de los Estados, así como el contexto institucional y la interacción de los jugadores existentes en el sistema político de cada realidad nacional. Este estudio se ha caracterizado por la diversidad de enfoques teóricos (Theodolou y Cahn, 1995) desde los modelos de proceso, sistémicos, a los modelos centrados en actores (Moran et al., 2007).

Por otro lado, desde la ciencia política podríamos decir que las políticas públicas estudian una dimensión más acotada del poder, aquella relacionada con el resultado de la lucha por el poder en una dimensión concreta y específica como son las acciones y omisiones del Estado en problemas concretos que afectan a las sociedades complejas y democráticas en el mundo contemporáneo. Los diferentes enfoques que dominan la ciencia política contemporánea, como el institucionalismo y la teoría de elección racional y aquellas miradas de la política desde la perspectiva de la economía han colaborado también al análisis de las políticas públicas en diferentes contextos históricos y nacionales.

Desde que el concepto fue acuñado en la década de los cincuenta, las políticas públicas han tenido un desarrollo vertiginoso, especialmente en Estados Unidos. Cientos de universidades desarrollan estudios de policy analysis, existiendo cursos de pregrado y posgrado que estudian las políticas públicas (principalmente a nivel

4 La literatura sobre políticas públicas ha desarrollado toda un área de investigación respecto al concepto de problemas en políticas públicas. 
de doctorado). El analista de políticas es una profesión que tiene ocupación tanto dentro del Estado como fuera de él en Estados Unidos.

Como suele suceder en las ciencias sociales no existe una única definición de políticas públicas, aunque la mayoría concuerda o tiene en común definir las políticas públicas como las acciones (o inacciones) del Estado para solucionar ciertos problemas públicos. Tampoco parece haber una única definición de política (policy). Aguilar (2006) la define como "un comportamiento propositivo, intencional, planeado, no simplemente reactivo, casual. Se pone en movimiento con el objetivo de alcanzar ciertos objetivos a través de ciertos medios: una acción con sentido" (Aguilar, 2006:24). Por otro lado, lo público o pública (public) se refiere al espacio colectivo en que se discuten los aspectos que influyen a la vida en sociedad. Más que todo el concepto de public se entiende en oposición a lo privado. Es decir, a políticas desarrolladas por el sector privado o agentes no estatales para el mundo privado (por ejemplo, las diferentes políticas de una empresa).

Los autores subrayan diferentes énfasis a la hora de definir el concepto de política pública. Una parte de estas definiciones llevan de manera más explícita una lógica normativa, como la del mismo Lasswell (1951) o la de Lahera (2008) que la vinculan con contextos democráticos. Otros enfatizan quién decide las políticas públicas (por ejemplo, la visión pluralista o de grupos -basada principalmente en Dahl (1967)-, la visión elitista (Mills, 1956), entre otras. Las políticas son el resultado de la interacción entre el Estado y la ciudadanía (Aguilar, 2006). Otras definiciones se centran en la acción concreta de los gobiernos argumentando que las políticas públicas son lo que los funcionarios públicos o el gobierno (por ende los ciudadanos representados en estos funcionarios) deciden o no hacer respecto a los problemas públicos (Kraft y Furlong, 2007).

El estudio de las policy sciences se ha destacado por tres aspectos: la estructuración de esta literatura orientada a los problemas; el enfoque multidisciplinario de esta disciplina, y la necesidad de vincular este estudio con opciones valóricas. Es decir, conocimiento para el mejoramiento de la democracia y dignidad humana (De León y Vogenbeck, 2007).

Metodológicamente, las políticas públicas pueden ser analizadas con diferentes herramientas (Bracho, 2010). Los métodos cuantitativos aplicados a las políticas públicas permiten generar información tanto para el decision maker como para los 
académicos e investigadores (Yang, 2007). Por otro lado, la metodología cualitativa permite profundizar las particularidades de las diferentes políticas en contextos específicos o conocer temas particulares respecto a políticas públicas singulares (Yanow, 2007; Sadovnik, 2007). En el primer caso, los métodos cuantitativos han estado vinculados históricamente a la economía, mientras que los cualitativos a los estudios provenientes de otras ciencias sociales. Por otro lado, dada la particularidad de las decisiones adoptadas para solucionar los problemas públicos, el estudio de caso es una forma de investigación muy extendida en el tema de las políticas públicas (Borges, 1995).

Precisamente, la utilización de herramientas de carácter cualitativo sirve de base a académicos y políticos para criticar la capacidad que tiene el enfoque de política pública para generar conocimiento teórico caracterizado por su generalización y abstracción desde las ciencias sociales, particularmente los estudios de caso (De León y Vogenbeck, 2007).

Por otro lado y sin miedo a exagerar, el ámbito que ha sido más utilizado del enfoque de políticas públicas ha sido el modelo del ciclo de políticas. Este modelo divide a las políticas públicas en tres partes: diseño, implementación y evaluación (Sabatier, 1999; Jann y Wegrich, 2007)5.

El diseño de políticas es aquella etapa en la cual se analiza la selección de una determinada política pública. La implementación se centra en la gestión o aplicación de esta política. Finalmente la evaluación es información sobre la implementación que permite generar nuevas decisiones sobre la mantención o rediseño de la política en cuestión. A la vez este esquema ha sido criticado por su debilidad teórica y empírica (Jann y Wegrich, 2007). El modelo de proceso de políticas se insertó en un comienzo dentro del estudio más general del proceso de toma de decisiones, especialmente el vinculado al diseño de las políticas. A partir de los setenta (Pressman y Wildavsky, 1984) el tema de la implementación se comenzó a observar como un ámbito separado del de diseño (Pressman y Wildavsky, 1984; Mazmanian y Sabatier, 1989). Los estudios de implementación estuvieron centrados primeramente en un modelo más centralizado como el top-down, para luego pasar a uno contrario, como el bottom-up. El estudio de la evaluación de

5 Existen otros modelos de etapas que incluyen un mayor número de éstas, como el del propio Lasswell. 
políticas vino a complementar los anteriores (Fischer, 1995; Vedung, 2005), siendo en general, el menos desarrollado, especialmente en América Latina y Chile. Con el tiempo la literatura ha convenido en señalar que el modelo del ciclo de política es útil para distinguir las etapas de una política pero no da cuenta de la realidad, en que muchas veces las políticas están en constante revisión (De León y Vogenbeck, 2007). Este modelo de ciclo, además, es el que tiene más aplicación práctica en la discusión gubernamental y pública.

Posterior al modelo de ciclo de políticas se desarrollaron otras áreas de investigación en políticas públicas. Por ejemplo, el tan conocido y utilizado concepto de incrementalismo en políticas públicas (Lindblom, 1959). En la década del sesenta se desarrollaron numerosos estudios sobre el proceso de agenda-setting (Cobb y Elder, 1983; Kingdon, 1984; Baumgartner y Jones, 1993; McComb, Shaw y Weaver, 2009), definido como aquel en que un problema es seleccionado para ser sujeto de acción por parte del Estado. La base de este análisis fue que la selección de un problema es un proceso político. Perspectivas más académicas como las sostenidas por Fischer (2007) o Stone (1997) abordan un análisis de política pública más centrado en lo teórico y normativo ${ }^{6}$. También se han desarrollado un sinnúmero de manuales (Pérez Sánchez, 2006; Kraft y Furlong, 2007) y handbooks (Moran et al., 2006; Fischer et al. 2007).

Por otro lado, existe una dimensión práctica del análisis de políticas públicas o policy analysis (Weimer y Vining, 1991). Un ejemplo lo constituyen las recomendaciones desarrolladas por Bardach (2005), quien postula un conjunto de pasos para realizar un exitoso análisis de política.

Desde la perspectiva de la ciencia política, el análisis de las políticas públicas se ha vinculado con el análisis de actores, reglas del juego y acciones. Generalmente las políticas públicas son consideradas como variables dependientes (Madrid, 2003). Por ejemplo, la literatura sobre modelos de capitalismo (models of capitalism) analiza las diferentes políticas que conforman los diferentes tipos de capitalismo (Castiglioni, 2002; Huber, 2003).

6 Stone (1997), por ejemplo, basa su argumento en la crítica al modelo racional de políticas públicas. 


\subsection{Políticas públicas en América Latina}

Autores latinoamericanos, entre ellos Cabrero (2000), se preguntan hasta qué punto "viaja" o no el enfoque de políticas públicas a distintos contextos políticos, culturales, históricos, económicos, diferentes a los teorizados por el modelo norteamericano. El autor ejemplifica su argumento centrándose en el Estado mexicano y su relación con el contexto histórico-político. El carácter particular del proceso de políticas hace que sea especialmente importante analizar el contexto en que se dan estas acciones gubernamentales.

Por otro lado, al igual que Cabrero (2000), otros autores enfatizan el contexto histórico para destacar las particularidades de las políticas públicas en América Latina. Sojo Garza-Adalpe (2006) sostiene que existen condiciones históricas y políticas en el caso mexicano y en América Latina en general que son necesarias para analizar las políticas públicas: transiciones a la democracia, sistemas políticos, electorales, etc. Por otro lado, hay ciertos aspectos específicos de los Estados latinoamericanos que le confieren singularidad al proceso de políticas públicas. Por ejemplo, la relación del Estado con los partidos, la relación Estado-sociedad, la menor institucionalización o la influencia de las instituciones informales. Para Latinoamérica en general y Chile en particular surge también la pregunta sobre el mecanismo a través del cual se expande o difunde el enfoque de políticas públicas, si es un efecto de la modernización, quiénes y cómo difunden este conocimiento y quiénes son los agentes modernizadores (por ejemplo organismos internacionales y élites vinculadas a éstos).

Relacionado con lo anterior, otra de las novedades en relación a la utilización del enfoque de políticas públicas, es que éste ha sido adoptado por los organismos internacionales como el Banco Interamericano de Desarrollo (BID), el Fondo Monetario Internacional (FMI) y el Banco Mundial (BM). Estos han dado numerosas recetas de políticas que se relacionan con la implantación y consolidación en la región del modelo de libre mercado a través del diseño y evaluación de políticas públicas específicas (por ejemplo, educación, pensiones, entre otras). Recientemente, organismos como el BID han valorado los factores políticos como fundamentales a la hora de explicar la variación del policy outcome en políticas públicas en la región (Stein et al., 2006). Vemos entonces cómo este enfoque, fortalezas más o menos, se ha convertido en un enfoque que permite discutir globalmente 
las acciones del Estado, como crisis económicas, déficit fiscal, reformas a la salud, educación, entre otros, pero todos bajo un mismo paradigma económico.

En el entendimiento que el enfoque de políticas públicas -al menos en el caso latinoamericano- está ligado a cierto modelo de desarrollo en que el manejo estable de los recursos (a nivel macroeconómico y microeconómico) es fundamental. Es en este sentido que la economía se inserta en el último tiempo como la disciplina del conocimiento experto. Esto tiene su correlato institucional en algunos países con la importancia dada a los ministerios de Hacienda, como es el caso de Chile y México.

\subsection{Políticas Públicas en Chile: experticia y economía}

Como señalamos en la introducción, el uso del concepto de políticas públicas comenzó a ser utilizado de manera masiva en Chile a partir de los años noventa. Ahora bien, no ha sido hasta los últimos años que el término ha sido masificado en el discurso público.

Vinculado a lo anterior, parte de la discusión ha estado centrada en el rol que han tenido los tecnócratas o expertos en el diseño y evaluación de políticas públicas. Estos tecnócratas han tenido un rol clave en la aplicación de políticas públicas especialmente en el ámbito macroeconómico.

Ahora bien, queda por definir qué es la tecnocracia, qué son los tecnócratas. Existe acuerdo en que la tecnocracia es un fenómeno político, pues éste está relacionado con quién decide, quién finalmente influye en los asuntos públicos, en las políticas, y en una dimensión más general, en el poder. En otras palabras, los tecnócratas ocupan espacios de decisión tradicionalmente ocupados por políticos. Desde su origen, se la ha visto opuesta a la democracia por la naturaleza no electa de estos actores (Meynaud, 1968; Fischer, 1990; Centeno, 1994). Entonces la pregunta general que está detrás de esta discusión es cuál es el rol de la experticia en las decisiones públicas que tradicionalmente han ocupado los políticos.

Esta discusión forma parte de otra más general y de larga data que se vincula con la tensión existente entre políticos y burócratas (Aberbach, Putnam y Rockman, 1981). Si bien esta literatura se ha desarrollado especialmente en democracias del mundo desarrollado, no es menos cierto que el fenómeno tecnocrático viene 
a complejizar la relación entre los diferentes actores del proceso de decisión y administración del Estado y los gobiernos. Para el caso chileno un análisis que se centra en este tipo de óptica es el de Cleaves (1974), quien analiza un caso particular como el Ministerio de Vivienda. Cleaves sostiene que en esta relación entre burocracia y políticos, los segundos dominan a los primeros. Similar argumento sostiene Valenzuela (1984), quien analizó el caso del higher civil service en Chile. Este autor sostiene que si bien este servicio tiene capacidad profesional, ésta no es suficiente, pues para acceder a estos cargos es necesario tener credenciales políticas.

Dávila (2010) señala tres características en relación a la literatura sobre tecnocracia: la primera, y sobre la que parece haber acuerdo, es que ellos basan su influencia en su experticia mientras que los políticos lo hacen en su experiencia en las elecciones competitivas (Fischer, 1990; Centeno y Silva 1998); la segunda, sobre la cual también parece existir acuerdo, se relaciona con el nivel de influencia de estos actores de la elite de gobierno. La literatura diferencia a los tecnócratas de los técnicos por el nivel de influencia de los primeros en el proceso de toma de decisiones (Meynaud, 1968). Los técnicos son expertos bajo la dirección de otras élites, mientras que los tecnócratas son actores que tienen acceso a decisiones que van más allá de su área de experticia y que generalmente han sido monopolio de los políticos. Finalmente, una tercera característica de esta literatura es que no existe una única definición de tecnócratas (Centeno, 1998; Silva, 1991).

Algunos autores centran sus definiciones en la trayectoria académica de los tecnócratas para poder caracterizarlos (Ai Camp, 1985), otros en las características de sus decisiones (Fischer, 1990) y otros los relacionan con determinadas ideologías en particular (Teichman, 2001). Por su parte, Dávila (2010) sostiene que en cuanto a la profesión de los tecnócratas, no existe acuerdo respecto a qué profesiones se vinculan con la tecnocracia.

Hay quienes argumentan que los tecnócratas provienen de las ciencias sociales duras como negocios, administración y leyes (Ross Schneider, 1998), otros los vinculan especialmente con la profesión de economistas (Ai Camp, 1983; Montecinos y Markoff, 1993; Dávila, 2010). Silva (2009) argumenta que si bien la mayoría de los tecnócratas son ingenieros, economistas, expertos financieros o administradores, personas con educación formal en sociología y ciencia política 
pueden ser tecnocratizados y aceptar que las decisiones pueden ser tomadas por expertos. Montecinos (1998) relaciona la introducción de reformas estructurales con la influencia tecnocrática y la creciente influencia de los economistas. Dávila $(2010,2011)$ asume lo señalado por parte de la literatura en cuanto a la importancia de la economía como profesión que distingue a los tecnócratas. En el caso chileno, más que ninguna otra profesión, la de economista es la que más se repite en quienes ejercen la tecnocracia, es decir quienes ocupan cargos de influencia política y de políticas (como el de ministro) basados no sólo en su trayectoria política, sino que principalmente, en su experticia técnica.

Como ya señalamos, los tecnócratas (vinculados a la economía) aparecen vinculados con la introducción de reformas neoliberales (Teichman, 2001), por un lado, y con la administración macroeconómica estable en contextos de economías de libre mercado, por otro -como lo es en el caso chileno- (Dávila, 2011).

Vemos entonces cómo la economía, como disciplina del conocimiento, se vincula con estabilidad en las políticas y el manejo del gobierno. Si antes el cambio era el concepto que guiaba el ritmo de evolución (vinculado a la instalación de un nuevo proyecto político de desarrollo), podemos argumentar que a partir de 1990 la estabilidad es el ritmo valorado desde la perspectiva de gobernar al no haber diferentes modelos de desarrollo en juego.

Como se señaló previamente, para el caso chileno también los tecnócratas se relacionan con la economía. Se argumenta que el rol de los economistas en el proceso de toma de decisiones ha aumentado desde que el primer economista ocupó la Dirección de Presupuesto durante el gobierno de Alessandri (Markoff y Montecinos, 1993). Se añade además que los economistas no sólo han penetrado el Estado sino también los partidos políticos. Los economistas dieron a los partidos de oposición a la dictadura un nuevo discurso. Como señala Montecinos (1998), el despliegue de economistas indicó la conformidad de los partidos con algunos de los rituales nuevos, que presuntamente garantizaban legitimidad política, como el interés por la eficiencia, la estabilidad macroeconómica y la competitividad internacional. Lo anterior explicaría la influencia que tuvieron los tecnócratas y el espacio que fue cedido por los políticos tradicionales dentro de los partidos. Además se argumenta que existe relación entre la presencia tecnocrática y la necesidad de disminuir la inestabilidad política (como los períodos entre 1931 y 1938 y en- 
tre 1988 y 1990 (Silva, 2006). Por otro lado, Silva (2009) afirma que la presencia de la tecnocracia en el caso de la Concertación se debe a la necesidad de mantener el modelo económico y por el proceso de aprendizaje político vivido por la élite. Como señalamos, para Silva los tecnócratas pueden ser economistas, ingenieros, abogados y también sociólogos y cientistas políticos que se hayan tecnocratizado.

Otros estudios analizan la influencia tecnocrática en el diseño de políticas públicas. Por ejemplo, Olavarría et al., (2011), quienes, a través de un estudio de caso con metodología cualitativa, analizan el rol de los tecno-políticos en el diseńo de políticas relacionadas con la modernización de la gestión pública. Estos autores argumentan la importancia de este actor en el diseño de estas políticas.

A partir de lo anteriormente señalado se ha considerado que en el caso chileno es esperable que el valor dado al conocimiento experto en economía tenga un correlato en cuanto al valor que se le asigna a éste en la formación de expertos de políticas públicas. Es decir, debería haber una cierta coherencia entre el valor dado al conocimiento experto en economía dado por las elites de gobierno y políticas en general y la concentración de estudios de políticas públicas en áreas vinculadas con escuelas de economía.

Un buen ejemplo de lo anterior sería el caso de la Corporación de Estudios para América Latina (CIEPLAN), que tuvo una influencia determinante en la formación de equipos y políticas públicas en el primer gobierno de la Concertación (1990-1994) y que actualmente concentra a importantes economistas y tecnócratas de esta coalición de gobierno que ocupó el poder en Chile durante veinte años.

Para más adelante queda la pregunta de cuán masificado está este enfoque en el Estado chileno. Como señala Dávila $(2010,2011)$ para el caso de los gobiernos concertacionistas, la influencia del conocimiento tecnocrático fue intensiva más que extensiva, en el sentido de que tuvo una presencia constante en Hacienda y menor en el resto de los ministerios (análisis sólo del nivel de ministros y subsecretarios). 


\section{ASPECTOS METODOLÓGICOS}

Como concepto operativo, definiremos políticas públicas como las acciones del Estado para solucionar un problema definido como público.

Esta definición operativa se centra en el enfoque de procesos y/o de propuesta de política conocido como etapa de diseño. Modelo cuestionable en torno a su lógica de causa efecto, pero que para términos operativos permite controlar de algún modo un proceso considerado como infinito.

Como se ha mencionado, el objetivo de este artículo es hacer un primer reconocimiento de lo que se está pensando y enseñando sobre políticas públicas en Chile hasta el 2010 y poder establecer si el valor asignado a la economía (a través de los tecnócratas) como uno de los conocimientos expertos más importantes, se relaciona con la enseñanza de políticas públicas en Chile. Para este fin, se realiza una revisión exhaustiva de todas las mallas de carreras de pregrado en Chile, para construir nuestra muestra en la cual consideramos sólo aquellas que tengan al menos un ramo de políticas públicas. En el caso de los posgrados, se seleccionaron sólo aquellos que se denominen orientados fundamentalmente a las políticas públicas o con mención en políticas públicas. Toda la revisión fue realizada en el mes de enero de 2011, considerando programas nuevos y antiguos que aún se dicten.

\section{ANÁLISIS}

En esta sección se analizan los datos obtenidos del trabajo de campo (ver anexo 1). Del análisis de éstos podemos afirmar que a nivel de carreras de pregrado el estado de la enseñanza de las políticas públicas es el siguiente:

Veintiún universidades imparten cursos o carreras vinculadas a las políticas públicas, dieciséis están ubicadas en la Región Metropolitana. Este número indica que más de la mitad de las carreras con formación en políticas públicas, se encuentran en un espacio físico compartido con el gobierno central, desde donde emanan la gran mayoría de las políticas públicas para el país. Es decir, esto se condice con el grado importante de centralización que exhibe nuestro país no sólo en términos políticos y económicos, sino también culturales. 
Del total de carreras de pregrado, cuatro pertenecen a facultades de economía, administración, negocios o ingeniería. Un total de veinte y seis pertenecen a facultades vinculadas a las ciencias sociales, humanidades, gobierno o ciencias jurídicas, mientras que tres pertenecen a otro tipo de facultad o institutos o se encuentran fuera de la orgánica de una facultad. Es decir, a nivel de pregrado las políticas públicas están vinculadas a las ciencias sociales en general y no a la economía en particular.

Por otro lado, del total de carreras de pregrado once son de ciencia política, ocho de sociología, siete de administración pública, cinco de trabajo social, una de socio-economía y una de ingeniería en ejecución en gestión pública. Como vemos, mayoritariamente las carreras ligadas a las ciencias sociales son aquellas que imparten cursos de políticas públicas en pregrado, mientras que sólo una de ellas es vinculada a la ingeniería y una a un híbrido entre sociología y economía. Como es esperable en una disciplina a partir de la cual se discute el enfoque de políticas públicas en la década del cincuenta, ciencia política concentra la mayoría de los cursos en políticas públicas.

En una recolección de datos en relación a las escuelas de pregrado del área de la economía, administración o empresas, los cursos de políticas públicas dictados no figuran entre las líneas de formación principales de las mallas curriculares. En algunos casos aislados, los cursos de políticas públicas son mencionados, pero como electivos de perfeccionamiento o formación general, dictados por otras facultades, generalmente de ciencias sociales o administración pública. Otro dato importante es que a nivel de pregrado, las mallas curriculares que contemplan cursos de políticas públicas consideran cursos de economía como pre-requisitos para poder acceder a ellos. En el caso de las ciencias sociales, las escuelas de sociología generalmente tienen en sus mallas incorporados cursos relacionados con gestión pública, evaluación de proyectos, desarrollo local, entre otros.

Al nivel de los posgrados, encontramos dos tipos: diplomados o postítulos (ver anexo 2) y magíster (ver anexo 3). Por otro lado, para el caso chileno, a nivel de doctorado no existe oferta en el área de políticas públicas. Es decir, todavía en Chile no se aprecian estudios académicos especializados de políticas públicas como tales.

De los diplomados o postítulos, dos son dictados por la Facultad de Economía y Negocios de la Universidad de Chile. Mientras la Facultad de Arquitectura, 
Diseño y Estudios Urbanos de la Pontificia Universidad Católica de Chile (PUC) dicta un diplomado en políticas públicas ${ }^{7}$.

A nivel de magíster, son nueve los posgrados dictados actualmente en Chile. Cuatro de ellos pertenecen a facultades de ciencias sociales y gobierno, mientras que los otros cinco a facultades de economía, negocios, administración y matemáticas.

A la luz de los resultados, podemos observar que la oferta de posgrados enfocados en políticas públicas, pertenece en su mayoría a facultades de economía, administración y negocios, con un $58,3 \%$ de la oferta total. Mientras que las facultades de gobierno y ciencias sociales dictan el 33,3\% de la oferta de posgrado. El 8,3\% restante pertenece a facultad de arquitectura, diseño y estudios urbanos.

En relación a los centros de estudios vinculados a partidos políticos, podemos mencionar a los más destacados en políticas públicas y conocidos como "generadores de ideas". En este nivel encontramos cinco: el Instituto Igualdad vinculado al Partido Socialista, el Instituto Libertad con vínculo a Renovación Nacional, el partido Demócrata Cristiano y su vínculo con el Centro de Estudios del Desarrollo (CED), el Partido Por la Democracia con vinculación a Chile 21 y finalmente Libertad y Desarrollo con la Unión Demócrata Independiente. En estos centros de estudio el enfoque de políticas públicas varía en complejidad, concentrándose, como lo señalamos en el análisis del diseño de políticas públicas, con mayor o menor grado de profundidad. Generalmente estos centros de estudios están dedicados a formar cuadros profesionales (en menor medida) y a la preparación de proyectos de ley y asesorías parlamentarias (en mayor medida) (Guzmán, 2010).

De los centros ya mencionados, dos de ellos son abiertamente reconocidos por su ligazón con los partidos de la coalición gobernante, y por ser formadores no sólo de ideas, sino que también de capital humano para nutrir a los altos cargos de la administración pública. Estos son: el Instituto Libertad de Renovación Nacional, y la Fundación Libertad y Desarrollo, perteneciente al partido Unión Demócrata Independiente. De manera intencional dejamos fuera la Fundación Jaime Guzmán, por tener vinculación con temas más ideológicas y de formación partidaria

A pesar de ser un posgrado impartido por una Facultad de Arquitectura, Diseño y Estudios Urbanos, los profesores que en él imparten cátedra son pertenecientes a distintas facultades de la Universidad. A pesar de este carácter multidisciplinario, para simplificar el análisis decidimos considerar solamente la Facultad de la que proviene, y no en función de quienes imparten cursos en él. 
de la UDI, punto que no deja de ser interesante, pero que se aleja de los focos de nuestro estudio.

Algunos de estos centros de estudios fueron creados en plena dictadura, como el CEP y CED, ambos creados en 1980. El Instituto Libertad y Desarrollo y el Instituto Libertad fueron creados en 1990. De todos estos el Instituto Libertad y Desarrollo y el CED utiliza la palabra políticas públicas para referirse a su misión institucional. El resto menciona el concepto de temas públicos, sin aludir específicamente a éste.

El Instituto Igualdad, vinculado al Partido Socialista, sirve también como generador de ideas y políticas, como también lo hace Chile 21 para el caso del Partido por la Democracia. En el caso de los dos espacios mencionados, funcionan además como plataformas programáticas para los líderes partidarios y formación ideológico-técnica de sus militantes.

En la lista figuran adicionalmente dos centros de estudios y generación de políticas públicas, y considerados como centrales en el sistema político chileno, como lo son el Centro de Estudios Públicos (CEP-Chile) y la Corporación de Estudios para Latinoamérica (CIEPLAN). En ambos casos, no se reconoce un vínculo directo con ningún partido político, pero en ambos casos es posible identificar un nexo con coaliciones o posturas ideológicas. El CIEPLAN ha sido históricamente ligado a la Concertación, dado que muchos ex ministros o figuras importantes de sus gobiernos han trabajado o trabajan en él, en el rol de diseñadores o pensadores de políticas. Si bien el CEP-Chile no posee un nexo tan evidente, es considerado como un centro de pensamiento más ligado a un liberalismo de derecha.

En cuanto a los centros vinculados a las universidades destacan dos: el Instituto Nacional de Asuntos Públicos (INAP) dependiente de la Universidad de Chile y el Instituto de Políticas Públicas de la UC; ambos publican documentos y al mismo tiempo ofrecen cursos de administración pública el primero y diplomados en políticas públicas ambos. En el caso de estos dos centros de estudios, al encontrarse ligados a universidades, conservan la línea de las mismas, sin tener pensamientos de tipo político partidario establecidos ${ }^{8}$.

8 Si bien no se puede establecer un vínculo ideológico claro, el Centro de Política Públicas de la UC, ha sido ligado a la administración del Presidente Piñera como asesor en el área de políticas públicas. 


\section{CONCLUSIONES}

De acuerdo a los datos antes presentados, podemos argumentar que nuestra hipótesis de trabajo (la economía es la disciplina que domina el enfoque de políticas públicas), es razonablemente cierta en gran parte de los aspectos antes analizados. El enfoque de políticas públicas efectivamente se encuentra generalizado por parte de las ciencias sociales a nivel de pregrado, pero la enseñanza de experticia en políticas públicas se encuentra vinculada a las facultades de economía, administración o negocios.

En este sentido, nuestra tesis inicial a partir de la cual afirmábamos una predominancia de la economía en el proceso decisorio puede evidenciarse a partir de la oferta de posgrados en Chile. Esto es, los alumnos de distintas carreras vinculadas generalmente a las ciencias sociales son instruidos en cuestiones básicas del enfoque de políticas públicas, sus métodos y conceptos, mientras que quienes decidan especializarse en esas áreas deben hacerlo (para el caso chileno) desde el paradigma economicista. En términos simples, cuestiones de costo-beneficio terminan siendo predominantes en la creación de los futuros decision makers. Es decir, la lógica de la economía, centrada en el análisis de los recursos, de criterios como eficiencia y eficacia terminan imponiéndose en el análisis de las políticas públicas sobre otras aproximaciones a los temas públicos. El análisis económico es, entonces, el que predomina en el estudio de los temas públicos vinculados a las políticas públicas.

En relación a los centros de estudios más importantes a nivel país, están vinculados a partidos políticos, pero también funcionan como centros de formación programática-ideológica. Por lo tanto, se mueven en la dicotomía de la experticia técnica y el rol político-ideológico. En general, el enfoque de políticas públicas no está extendido, más en cuestiones formales de declaraciones de principios, sin llegar a profundizar en estudios disponibles para la ciudadanía. En este caso no descartamos el rol de asesoramiento que juegan con los parlamentarios de los partidos a los cuales están ligados, pero que los convierten en cuestiones más bien contingentes que continuas.

Otro punto importante de recalcar, es que hasta el año 2010, si bien estos centros de pensamientos estaban íntimamente ligados a los partidos políticos, las políticas 
públicas se pensaban "desde fuera" del gobierno, pero se ejecutaban desde dentro. Es decir, seguían siendo los ministerios los principales "hacedores" o "ejecutores" de políticas. Con el cambio de coalición podemos tener algunas luces de cambios con respecto a ello, por cuanto muchos de los investigadores ligados a centros de estudios de RN o la UDI, pasaron a formar parte de los equipos asesores ministeriales a ocupar cargos de confianza desde donde proyectan llevar a cabo muchos de los proyectos que llevan tiempo gestándose desde los centros de estudios.

En relación a todo lo antes mencionado, podemos afirmar que no hay evidencia concluyente para sostener que la economía haya permeado todo el conocimiento y enseñanza sobre políticas públicas, pero sí aquellos ámbitos de educación más especializados donde se analizan los temas públicos.

Para finalizar, esta investigación forma parte de una pregunta más general, que ya mencionamos al comienzo, y que se relaciona con el rol del conocimiento experto en las decisiones públicas en las democracias contemporáneas. Lo anterior está vinculado, además, con el rol de los partidos políticos y los políticos en estas decisiones. Qué tan expertas pueden ser las decisiones de política pública en Chile hoy. Cuál es la esfera o importancia del conocimiento experto en las decisiones de políticas públicas. Son los políticos o estos tecnócratas quienes deciden. En un mundo del conocimiento donde éste es cada vez más especializado, la pregunta es qué rol les cabe a los ciudadanos y sus organizaciones en este debate y cómo los partidos y sus políticos integran este conocimiento experto, o si, por el contrario, los partidos se divorcian de éste y pierden capacidad de debate en estos términos, centrándose en lenguajes más populistas o demagógicos.

\section{REFERENCIAS BIBLIOGRÁFICAS}

Aberbach, Joel D.; Putnman, Robert D.; Rockman, Bert A. (1981). Bureaucrats and Politicians in Western Democracies. Cambridge, MA: Harvard University Press.

Aguilar Villanueva, Luis (2006). La Hechura de las Políticas Públicas. México: Miguel Ángel Porrúa Ediciones.

Ai Camp, Roderic (1983). “El Tecnócrata en México”. Revista Mexicana de Sociología Vol. 45, №2, pp. 579-599. 
Ai Camp, Roderic (1985). "The Political Technocrat in Mexico and the Survival of Political System”. Latin American Research Review Vol. 20, N¹, pp. 97-117.

Altman, David (2000). "The politics of coalition formation and survival in multiparty presidential democracies. The Case of Uruguay, 1989-1999”. Party Politics Vol. 6, º3, pp. 259-283.

Amorim Neto, Octavio (2003). Cabinet Formation in Presidential Regimes. Paper prepared for the American Political Science Association. Philadelphia.

Amorim Neto, Octavio (2006). “The Presidential Calculus. Executive Policy Making and Cabinet Formation in the Americas". Comparative Political Studies Vol 39, № 4, pp. 415- 440.

Andrews, Clinton (2007). "Rationality in Policy Decision Making”. En: Fischer, F; Miller, G. y Sidney, M. Handbook of Public Policy Analysis. Theory, Politics and Methods. Boca Ratón: CRC Press.

Bardach, Eugene (2005). Practical Guide for Policy Analysis. The Eightfold Path to More Effective Problem Solving. Washington: Congressional Quarterly Inc.

Baumgartner, Frank y Jones, Bryan D. (1993). Agendas and Instability in American Politics. Chicago: The University of Chicago Press.

Borges, Ramón (1995). "El Estudio de Caso como Instrumento Pedagógico y de Investigación en Políticas Públicas" Estudio de Caso №4.

Bracho, Teresa (2010). "Políticas Basadas en Evidencia. La Política Pública como Acción Informada y Objeto de Investigación”. En: Merino, Mauricio, et al. Problemas, Decisiones y Soluciones. Enfoques de Política Pública. México. CIDE-EFE.

Cabrero, Enrique (2000). “Usos y Costumbres en la Hechura de las Políticas Públicas en México. Límites de las policy sciences en contextos cultural y políticamente diferentes”. Revista Gestión y Política Pública, Vol. IX, $N^{\circ} 2$. pp. 189-229.

Castiglioni, Rossana (2002). "Reformas a las Pensiones en América Latina: Orígenes y Estrategias". Revista Ciencia Politica, vol. XXV, N², pp. 173-189.

Centeno, Miguel Ángel (1994). Democracy within Reason. Technocratic Revolution in Mexico. University Park: Pennsylvania State University Press.

Centeno, Miguel Ángel y Silva, Patricio (1998). The Politics of Expertise in Latin America. New York: St. Martin's Press.

Chasquetti, Daniel (2006). "La Supervivencia de las Coaliciones Presidenciales de Gobierno en América Latina”. Revista PostData N¹1, pp. 163-192.

Cleaver, Peter (1974). Bureaucratic Politics and Administration in Chile. Berkeley: University of California Press. 
Cobb, Roger y Elder, Charles (1983). Participation in American Politics: the dynamics of Agenda Building. Baltimore: Johns Hopkins University Press.

Dahl, Robert (1967). Pluralist Democracy in the United States. Chicago: Rand McNally.

Dávila, Mireya (2010). “Tecnocracia y Democracia en el Chile Contemporáneo: el caso de los gobiernos de la Concertación (1990-2010)”. Revista de Sociología N²4, pp. 119-211.

Dávila, Mireya (2011). Governing Together: The Concertación Administrations in Chile (1990-2009). Chapel Hill, NC. University of North Carolina-Chapel Hill.

De León, Peter; Vogenbeck, Danielle (2007). “The Policy Sciences at the Crossroads”. En: Fischer, F; Miller, G. y Sidney, M. Handbook of Public Policy Analysis. Theory, Politics and Methods. Boca de Ratón: CRC Press.

Fischer, Frank (1990). Technocracy and the politics of expertise. Newbury Park: Sage Publications.

Fischer, Frank (1995). Evaluating Public Policy. Chicago: Nelson-Hall.

Guzmán, Gabriel (2010). Los partidos politicos como agentes de formulación de políticas: el caso del PS y el PRSD en Chile, 1990-2010. Ponencia presentada en el IX Congreso de Ciencia Política. Santiago, Chile.

Huneeus, Carlos (1998). "Tecnócratas y políticos en un régimen autoritario. Los Odeplan Boys y los Gremialistas en el Chile de Pinochet”. Revista Ciencia Política. Vol. XIX, №2, pp. 125-158.

Jann, Werner y Wegrich, Kal (2007). "Theories of the Policy Cycle”. En: Fischer, F.; Miller, G. y Sidney, M. Handbook of Public Policy Analysis. Theory, Politics and Methods. Boca Ratón: CRC Press.

Kingdon, John (1984). Agendas, Alternatives and Public Policies. New York: Addison-Wesley Educational Publishers.

Kraft, M. y Furlong, Scott (2007). Public Policy: Politics, Analysis and Alternatives. Washington, DC: CQ Press.

Huber, Evelyne (2003). Models of Capitalism, Lessons for Latin America. University Park: The Pennsylvania State University.

Lahera, Eugenio (2008). Introducción a las Politicas Públicas. Santiago: Fondo de Cultura Económica.

Lasswell, Harold (1951). “The Policy Orientation”. En: Lasswell, Harold y Lerner, Daniel. The Policy Sciences. Palo Alto: Stanford University Press.

Lindblom, Charles (1959). “The 'Science' of Muddling Through”. Public Administration Review Vol.19 N'2, pp. 79-88. 
Mc Comb, Maxwell; Shaw, Donald y Weaver, David (2009). Communication and Democracy: Exploring the Intellectual Frontiers in Agenda-Setting Theory. New York: Routledge.

Madrid, R. (2003). Retiring the State. The Politics of Pension Privatization in Latin America and Beyond. Stanford, CA: Stanford University Press.

Mazmanian, Daniel A. y Sabatier, Paul A. (1989). Implementation and Public Policy. Landham, MD: University Press of America.

Merino, Mauricio (2010). “La Importancia de la Ética en el Análisis de las Políticas Públicas”. En: Merino, Mauricio (compositor). Problemas, Decisiones y Soluciones. Enfoques de Política Pública. México: Fondo de Cultura Económica.

Meynaud, Jean (1968). Technocracy. New York: Free Press.

Mills, Wright (1956). The Power Elite. Oxford: Oxford University Press.

Montecinos, Verónica; Markoff, John (1993). "Democrats and technocrats: professional economists and regime transitions in Latin America". Canadian Journal of Development Studies. $\mathrm{N}^{\circ} 1$, pp. 7-22.

Montecinos, Verónica (1998). Economists, Politics and the State: Chile: 1958-1994. Amsterdam: CEDLA.

Moran, Michael; Rein, Martin y Goodin, Robert (2006). The oxford handbook of public policy. Oxford: Oxford University Press.

Olavarría, Mauricio; Navarrete, Bernardo y Figueroa, Verónica (2011). “¿Cómo se formulan las políticas públicas en Chile? Evidencia desde un estudio de caso”. Política y Gobierno, Vol. 18, No1, pp. 109-154.

Pérez Sánchez, Margarita, ed. (2006). Análisis de Politicas Públicas. España. Universidad de Granada.

Pressman, J. y Wildavsky, A. (1984). Implementation. The Oakland Project. Berkeley, CA: University of California Press.

Ross Schneider, Ben (1998). "The Material Bases of Technocracy: Investor Confidence and Neoliberalism in Latin America”. En: Centeno, Miguel Ángel y Silva, Patricio (1998). The Politics of Expertise in Latin America. New York: St. Martin's Press. New York.

Sabatier, Paul (1999). Theories of the Policy Process. Boulder: Westview Press.

Savodnik, Alan (2007). "Qualitative Research and Public Policy". En: Fischer, F.; Miller, G. y Sidney, M. Handbook of Public Policy Analysis. Theory, Politics and Methods. Boca Ratón: CRC Press. 
Schneider, Anne y Ingram, Helen (2007). "Public Policy and Democratic Citizenship: What Kinds of Citizenship Does Policy Promote?” En: Fischer, F.; Miller, G. y Sidney, M. Handbook of Public Policy Analysis. Theory, Politics and Methods. Boca de Ratón: CRC Press.

Siavelis, Peter (2000). The President and Congress in Postauthoritarian Chile. Institutional Constraints to Democratic Consolidation. University Park: The Pennsylvania State University Press.

Silva, Patricio (1991). "Technocrats and Politics in Chile: From the Chicago Boys to CIEPLAN Monks". Journal of Latin American Studies Vol.23, N², pp. 385-410.

Silva, Patricio (2006). "Los Tecnócratas y la Política en Chile. Pasado y Presente”. En Revista de Ciencia Política. Vol. XXVI, N², pp. 175-190.

Silva, Patricio (2009). In the Name of Reason. Technocrats and Politics in Chile. Pennsylvania: The Pennsylvania State University Press, University Park.

Sojo Garza-Aldape, Eduardo (2006). Políticas Públicas en Democracia. México. Fondo Cultura Económica.

Stein, Ernesto; Tommasi, Mariano; Echebarría, Koldo; Lora, Eduardo; Payne, Mark (2006). La política de las políticas públicas: progreso económico y social en América Latina: Informe 2006. New York: BID. Editorial Planeta.

Stone, Deborah (1997). Policy Paradox, The Art of Political Decision Making. New York: W.W. Norton and Company.

Susskind, Lawrence (2006). “Arguing, Bargaining, and Getting Agreement”. En: Moran, Michael; Rein, Martin; Goodin, Robert. The Oxford Handbook of Public Policy. Oxford: Oxford University Press.

Teichman, Judith (2001). The Politics of Freeing Markets in Latin America. North Carolina: The University of North Carolina Press.

Theodolou, Stella y Cahn, Matthew (1995). Public Policy. The Essential Readings. New Jersey: Prentice Hall.

Valenzuela, Arturo (1984). "Parties, Politics, and the State in Chile: The Higher Civil Service". En: Suleiman, Ezra. Bureaucrats and Policy Making. A comparative Overview. New York: Holmes \& Meier.

Vedung, Evert (2005). Public Policy and Program Evaluation. New Jersey: Transaction Publishers.

Weimer, David y Vining, Aidan (1991). Policy Analysis, Concepts and Practice. New Jersey: Prentince Hall. 
Yang, Kaifeng (2007). "Quantitative Methods for Policy Analysis". En: Fischer, F.; Miller, G. y Sidney, M. Handbook of Public Policy Analysis. Theory, Politics and Methods. Boca Ratón: CRC Press.

Yanow, Dvora (2007). "Qualitative-Interpretive Methods in Policy Research”. En: Fischer, F.; Miller, G. y Sidney, M. Handbook of Public Policy Analysis. Theory, Politics and Methods. Boca Ratón: CRC Press.

\section{Sitios en internet ${ }^{9}$}

Sitio Web del Centro de Estudios Públicos (CEP-Chile) http:// www.cepchile.cl

Sitio Web del Instituto Igualdad http://www.igualdad.cl

Sitio Web del Instituto Libertad http://www.institutolibertad.cl

Sitio Web del Instituto Libertad y Desarrollo http://www.lyd.cl

Sitio Web Chile 21 http://www.chile21.cl

Sitio Web Centro de Estudios del Desarrollo http://www.ced.cl

Sitio Web Corporación de Estudios para Latinoamérica CIEPLAN-Chile http://www.cieplan.cl Sitios Web Ues Chile http://www.ues.cl

9 Todos los sitios fueron consultados entre octubre de 2010 y enero de 2011, fecha en la cual se realizó el trabajo de campo. 


\section{Anexo $\mathrm{N}^{\circ} 1$}

\section{Carreras de pregrado}

\begin{tabular}{|c|c|c|c|}
\hline UNIVERSIDAD & CARRERA & FACULTAD & RAMOS \\
\hline Universidad de Chile & $\begin{array}{l}\text { Administración } \\
\text { Pública }\end{array}$ & Instituto de Asuntos Públicos & Políticas Públicas \\
\hline Universidad de Chile & Sociología & Facultad de Ciencias Sociales & Políticas Públicas \\
\hline $\begin{array}{l}\text { Universidad de } \\
\text { Concepción }\end{array}$ & Ciencia Política & $\begin{array}{l}\text { Facultad de Ciencias Jurídicas } \\
\text { y Sociales }\end{array}$ & Políticas Públicas \\
\hline $\begin{array}{l}\text { Pontificia Universidad } \\
\text { Católica de Chile }\end{array}$ & Ciencia Política & $\begin{array}{l}\text { Facultad de Historia, } \\
\text { Geografía y Ciencia Política }\end{array}$ & $\begin{array}{l}\text { Formación de Políticas } \\
\text { Públicas }\end{array}$ \\
\hline $\begin{array}{l}\text { Pontificia Universidad } \\
\text { Católica de Chile }\end{array}$ & Trabajo Social & Facultad de Ciencias Sociales & $\begin{array}{l}\text { Trabajo Social y Políticas } \\
\text { Públicas }\end{array}$ \\
\hline $\begin{array}{l}\text { Pontificia Universidad } \\
\text { Católica de Chile }\end{array}$ & Sociología & Facultad de Ciencias Sociales & Políticas Públicas \\
\hline Universidad de Santiago & $\begin{array}{l}\text { Administración } \\
\text { Pública }\end{array}$ & $\begin{array}{l}\text { Facultad de Administración y } \\
\text { Economía }\end{array}$ & Políticas Públicas \\
\hline $\begin{array}{l}\text { Universidad de } \\
\text { Valparaíso }\end{array}$ & Socioeconomía & $\begin{array}{l}\text { Facultad de Ciencias } \\
\text { Económicas y Administrativas }\end{array}$ & Políticas Públicas \\
\hline $\begin{array}{l}\text { Universidad de } \\
\text { Valparaíso }\end{array}$ & Trabajo Social & $\begin{array}{l}\text { Facultad de Derecho y } \\
\text { Ciencias Sociales }\end{array}$ & $\begin{array}{l}\text { Estado y Políticas } \\
\text { Públicas } \\
\text { Taller de Políticas } \\
\text { Públicas. I, II, III }\end{array}$ \\
\hline $\begin{array}{l}\text { Universidad de } \\
\text { Valparaíso }\end{array}$ & $\begin{array}{l}\text { Administración } \\
\text { Pública }\end{array}$ & $\begin{array}{l}\text { Facultad de Ciencias } \\
\text { Económicas y Administrativas }\end{array}$ & Políticas Públicas \\
\hline $\begin{array}{l}\text { Universidad de La } \\
\text { Frontera }\end{array}$ & Trabajo Social & $\begin{array}{l}\text { Facultad de Educación y } \\
\text { Humanidades }\end{array}$ & Políticas Públicas \\
\hline $\begin{array}{l}\text { Universidad } \\
\text { Arturo Prat }\end{array}$ & Trabajo Social & $\begin{array}{l}\text { Facultad de Ciencias Sociales } \\
\text { y Humanidades }\end{array}$ & $\begin{array}{l}\text { Políticas Públicas y } \\
\text { Sociales en América } \\
\text { Latina y Chile }\end{array}$ \\
\hline $\begin{array}{l}\text { Universidad } \\
\text { Arturo Prat }\end{array}$ & Sociología & $\begin{array}{l}\text { Facultad de Ciencias Sociales } \\
\text { y Humanidades }\end{array}$ & $\begin{array}{l}\text { Políticas Públicas y } \\
\text { Sociales }\end{array}$ \\
\hline $\begin{array}{l}\text { Universidad Academia } \\
\text { Humanismo Cristiano }\end{array}$ & $\begin{array}{l}\text { Ingeniería de } \\
\text { Ejecución en } \\
\text { Gestión Pública }\end{array}$ & $\begin{array}{l}\text { Facultad de Gobierno y } \\
\text { Gestión Pública }\end{array}$ & Políticas Públicas \\
\hline $\begin{array}{l}\text { Universidad Academia } \\
\text { Humanismo Cristiano } \\
\end{array}$ & $\begin{array}{l}\text { Administración } \\
\text { Pública }\end{array}$ & $\begin{array}{l}\text { Facultad de Gobierno y } \\
\text { Gestión Pública }\end{array}$ & Políticas Públicas \\
\hline $\begin{array}{l}\text { Universidad Academia } \\
\text { Humanismo Cristiano }\end{array}$ & Ciencia Política & Facultad de Ciencias Sociales & Políticas Públicas \\
\hline $\begin{array}{l}\text { Universidad } \\
\text { Alberto Hurtado }\end{array}$ & Sociología & Facultad de Ciencias Sociales & $\begin{array}{l}\text { Políticas Públicas y } \\
\text { Sociales }\end{array}$ \\
\hline
\end{tabular}




\begin{tabular}{|c|c|c|c|}
\hline UNIVERSIDAD & CARRERA & FACULTAD & RAMOS \\
\hline $\begin{array}{l}\text { Universidad } \\
\text { Alberto Hurtado }\end{array}$ & $\begin{array}{l}\text { Ciencia Política } \\
\text { y Relaciones } \\
\text { Internacionales }\end{array}$ & Facultad de Ciencias Sociales & Políticas Públicas \\
\hline $\begin{array}{l}\text { Universidad Católica } \\
\text { Cardenal Raúl } \\
\text { Silva Henríquez }\end{array}$ & Sociología & $\begin{array}{l}\text { Facultad de Ciencias Sociales, } \\
\text { Jurídicas y Económicas }\end{array}$ & $\begin{array}{l}\text { Estado y Políticas } \\
\text { Públicas }\end{array}$ \\
\hline Universidad Central & $\begin{array}{l}\text { Administración } \\
\text { Pública }\end{array}$ & $\begin{array}{l}\text { Facultad de Ciencia Política y } \\
\text { Administración Pública }\end{array}$ & $\begin{array}{l}\text { Formulación y Gestión } \\
\text { de Políticas Públicas }\end{array}$ \\
\hline Universidad ARCIS & Ciencia Política & $\begin{array}{l}\text { Facultad de Ciencias Sociales } \\
\text { y Humanidades }\end{array}$ & $\begin{array}{l}\text { Introducción a las P.P. } \\
\text { Modelos Teóricos de } \\
\text { Políticas Públicas } \\
\text { Taller de Análisis de } \\
\text { Políticas Públicas }\end{array}$ \\
\hline $\begin{array}{l}\text { Universidad del } \\
\text { Desarrollo }\end{array}$ & $\begin{array}{l}\text { Ciencia Política y } \\
\text { Políticas Públicas }\end{array}$ & Facultad de Gobierno & Políticas Públicas I, II \\
\hline $\begin{array}{l}\text { Universidad de } \\
\text { Viña del Mar }\end{array}$ & Trabajo Social & $\begin{array}{l}\text { Escuela de Ciencias Jurídicas } \\
\text { y Sociales }\end{array}$ & $\begin{array}{l}\text { Políticas Públicas y } \\
\text { Sociales }\end{array}$ \\
\hline $\begin{array}{l}\text { Universidad de } \\
\text { Viña del Mar }\end{array}$ & Sociología & $\begin{array}{l}\text { Escuela de Ciencias Jurídicas } \\
\text { y Sociales }\end{array}$ & Políticas Públicas \\
\hline $\begin{array}{l}\text { Universidad } \\
\text { Diego Portales }\end{array}$ & Sociología & $\begin{array}{l}\text { Facultad de Ciencias Sociales } \\
\text { e Historia }\end{array}$ & Políticas Públicas \\
\hline $\begin{array}{l}\text { Universidad } \\
\text { Diego Portales }\end{array}$ & Ciencia Política & $\begin{array}{l}\text { Facultad de Ciencias Sociales } \\
\text { e Historia }\end{array}$ & Políticas Públicas \\
\hline $\begin{array}{l}\text { Universidad } \\
\text { Gabriela Mistral }\end{array}$ & Ciencia Política & $\begin{array}{l}\text { Facultad de Humanidades y } \\
\text { Ciencias Sociales }\end{array}$ & Políticas Públicas \\
\hline $\begin{array}{l}\text { Universidad } \\
\text { Iberoamericana } \\
\end{array}$ & $\begin{array}{l}\text { Administración } \\
\text { Pública }\end{array}$ & $\begin{array}{l}\text { Facultad de Administración y } \\
\text { Negocios }\end{array}$ & Políticas Públicas I, II \\
\hline $\begin{array}{l}\text { Universidad } \\
\text { Miguel de Cervantes }\end{array}$ & $\begin{array}{l}\text { Ciencia Política } \\
\text { y Administración } \\
\text { Pública }\end{array}$ & No aplica & Políticas Públicas \\
\hline $\begin{array}{l}\text { Universidad } \\
\text { Andrés Bello }\end{array}$ & Sociología & Facultad de Ciencias Sociales & Políticas Públicas \\
\hline $\begin{array}{l}\text { Universidad } \\
\text { Andrés Bello }\end{array}$ & $\begin{array}{l}\text { Administración } \\
\text { Pública mención } \\
\text { Ciencia Política }\end{array}$ & $\begin{array}{l}\text { Facultad de Ciencias Jurídicas } \\
\text { y Sociales }\end{array}$ & Políticas Públicas \\
\hline $\begin{array}{l}\text { Universidad } \\
\text { San Sebastián }\end{array}$ & $\begin{array}{l}\text { Ciencias Políticas } \\
\text { y Gestión Pública }\end{array}$ & Facultad de Ciencias Sociales & Políticas Públicas \\
\hline $\begin{array}{l}\text { Universidad } \\
\text { Los Leones }\end{array}$ & $\begin{array}{l}\text { Ciencia Política } \\
\text { mención } \\
\text { Gobierno y } \\
\text { Relaciones } \\
\text { Internacionales }\end{array}$ & No aplica & Políticas Públicas \\
\hline
\end{tabular}




\section{Anexo $\mathrm{N}^{\circ} 2$}

\section{Postítulos en Políticas Públicas}

\begin{tabular}{lll}
\multicolumn{1}{c}{ UNIVERSIDAD } & \multicolumn{1}{c}{ FACULTAD } & \multicolumn{1}{c}{ DIPLOMADO } \\
\hline Universidad de Chile & Facultad de Economía y Negocios & Diploma en Public Policy \\
\hline $\begin{array}{l}\text { Universidad de Chile } \\
\text { Facultad de Economía y Negocios }\end{array}$ & $\begin{array}{l}\text { Diploma en Políticas Públicas en } \\
\text { Educación }\end{array}$ \\
\hline $\begin{array}{l}\text { Pontificia Universidad Católica } \\
\text { de Chile }\end{array}$ & $\begin{array}{l}\text { Facultad de Arquitectura, Diseño } \\
\text { y Estudios Urbanos }\end{array}$ & Diplomado en Políticas Públicas \\
\hline \hline
\end{tabular}

\section{Anexo $\mathrm{N}^{\circ} 3$}

\section{Magíster en Políticas Públicas}

\begin{tabular}{lll}
\multicolumn{1}{c}{ UNIVERSIDAD } & \multicolumn{1}{c}{ MAGÍSTER } & \multicolumn{1}{c}{ FACULTAD } \\
\hline Universidad de Chile & Políticas Públicas & Facultad de Economía y Negocios \\
\hline Universidad de Chile & Gestión y Políticas Públicas & $\begin{array}{l}\text { Facultad de Ciencias Físicas y } \\
\text { Matemáticas }\end{array}$ \\
\hline $\begin{array}{l}\text { Pontificia Universidad Católica } \\
\text { de Chile }\end{array}$ & $\begin{array}{l}\text { Magíster en Ciencia Política, } \\
\text { mención Políticas Públicas }\end{array}$ & $\begin{array}{l}\text { Facultad de Historia, Geografía y } \\
\text { Ciencia Política }\end{array}$ \\
\hline $\begin{array}{l}\text { Pontificia Universidad Católica } \\
\text { de Chile }\end{array}$ & $\begin{array}{l}\text { Magíster en Economía, mención } \\
\text { Políticas Públicas }\end{array}$ & Instituto de Economía \\
\hline Universidad de Santiago & $\begin{array}{l}\text { Magíster en Gerencia y Políticas } \\
\text { Públicas }\end{array}$ & $\begin{array}{l}\text { Facultad de Administración y } \\
\text { Economía }\end{array}$ \\
\hline Universidad Católica del Maule & $\begin{array}{l}\text { Magíster en Ciencias Sociales y } \\
\text { Políticas Públicas }\end{array}$ & Facultad de Ciencias Sociales \\
\hline Universidad Adolfo Ibáñez & $\begin{array}{l}\text { Magíster en Gerencia y Políticas } \\
\text { Públicas }\end{array}$ & Escuela de Gobierno \\
\hline Universidad Alberto Hurtado & $\begin{array}{l}\text { Magíster en Economía Aplicado a } \\
\text { Políticas Públicas }\end{array}$ & $\begin{array}{l}\text { Magíster en Economía y } \\
\text { Negocios }\end{array}$ \\
\hline Universidad del Desarrollo & \begin{tabular}{l} 
Magíster en Políticas Públicas \\
\hline \hline
\end{tabular} & \begin{tabular}{l} 
Facultad de Gobierno \\
\hline
\end{tabular}
\end{tabular}

